\title{
Estimación de las frecuencias alélicas y genotípicas de los genes CAPN1 Y CAST asociados a la calidad de la carne en bovinos de la Cuenca del Papaloapan
} Allelic and genotypic frequencies of CAPN1 and CAST, two genes associated to meat quality, in cattle from the Papaloapan Basin

C.M. Desgarennes-Alcalá1 ${ }^{1}$ S. del Moral $^{2}$, V.M. Meza-Villalvazo ${ }^{2}$, J.M. Peña-Castro² , J.P. ZárateMartínez $^{3}$ y J. Abad-Zavaleta ${ }^{2}$

Palabras Clave: bovinos de doble propósito; terneza de la carne; calpaína; calpastatina

\section{Recepción: 24-05-17 / Aceptación: 25-08-17}

\section{Resumen}

El ganado de doble propósito es aquel utilizado para producir carne y leche. Generalmente es el producto del cruce de razas de origen Cebú x europeas y/o Criollo. Este grupo genético se ha adaptado a las condiciones climáticas de temperatura, humedad, baja calidad de pastos y clases de parásitos que prevalecen en las regiones tropicales. En este trabajo, se realizó un escrutinio molecular para observar la frecuencia de dos polimorfismos del gen de la $\mu$-calpaína (CAPN1316, CAPN1-530) y uno del gen de la calpastatina (CAST), asociados a la suavidad de la carne en ganado bovino de doble propósito de la Cuenca del Papaloapan. Las pruebas se basaron en Reacción en Cadena de la Polimerasa (PCR) y Polimorfismos de la Longitud de Fragmentos de Restricción (RFLPs) con las enzimas de restricción Btg I, Ava II y Rsa I para los alelos C-G, A-G y C-G, respectivamente ( $\mathrm{n}=331)$. Para CAPN1-316 las frecuencias genotípicas obtenidas fueron de 0.03 (AA), 0.86 (GG) y 0.11 (GA) y las frecuencias alélicas fueron 0.86 (G) y 0.14 (A). Para el marcador CAPN1-530 las frecuencias genotípicas fueron de 0.02 (CC), 0.85 (GG) y 0.13 (GC), con una frecuencia alélica de 0.85 para G y 0.15 para C. Las frecuencias genotípicas para el marcador CAST fueron de 0.32 (CC), 0.28 (GG) y 0.4 (GC), con una frecuencia alélica de 0.68 para el alelo G y 0.32 para el alelo C. La población en estudio no se encontró en equilibrio Hardy Weinberg y los valores de $\chi^{2} \mathrm{P}$ con dos grados de libertad fueron de $0.964,0.985$ y 0.9803 para CAPN1-316, CAPN1-530 y CAST, respectivamente. De acuerdo con el porcentaje de la genotipificación de los marcadores en los genes CAPN1 y CAST se concluye que la población analizada tiene bajos índices de marcadores para la suavidad o terneza de la carne, probablemente por las cruzas no dirigidas que se realizan habitualmente, de esta forma se propone, mejorar la

${ }^{1}$ División de Estudios de Posgrado. Universidad del Papaloapan. E-mail: joeabad@ @otmail.com

${ }^{2}$ Instituto de Biotecnología. Universidad del Papaloapan

${ }^{3}$ Centro de Investigación Regional del Golfo, Instituto Nacional de Investigaciones Forestales, Agrícolas y Pecuarias

(C) Universidad De La Salle Bajío (México) 
calidad de la carne en base a programas de mejoramiento genético dirigido utilizando los animales genéticamente superiores resultantes de este estudio.

\section{Abstract}

The dual-purpose cattle are that used to produce meat and milk, is usually the product of miscegenation Cebu origin $\mathrm{x}$ European and / or creole. This genetic group has adapted to the climatic conditions of temperature, humidity, low quality pastures and any kind of parasite prevalent in tropical regions. In this work, a molecular thorough examination was performed to observe the frequency of two polymorphisms gene $\mu$-calpain (CAPN1-316, CAPN1-530) and one gene calpastatin (CAST), associated with the softness of meat cattle dual purpose of the Cuenca Papaloapan. The tests were based on polymerase chain reaction (PCR) and length polymorphisms Restriction Fragment (LPRF) with restriction enzymes Btg I, Ava II and Rsa I for CG, AG and CG alleles, respectively $(\mathrm{n}=331)$. To CAPN1-316 the genotypic frequencies obtained were 0.03 (AA), $0.86(\mathrm{GG})$ and $0.11(\mathrm{GA})$ and allele frequencies were $0.86(\mathrm{G})$ and $0.14(\mathrm{~A})$. CAPN1-530 marker for the genotype frequencies were 0.02 (CC), 0.85 (GG) and 0.13 (GC) with an allele frequency of 0.85 to 0.15 for $\mathrm{G}$ and $\mathrm{C}$. The genotype frequencies for the marker CAST were 0.32 (CC), 0.28 (GG) and 0.4 (GC) with an allele frequency of 0.68 for the $\mathrm{G}$ and 0.32 allele for allele C. The study population was found in Hardy Weinberg and values $\chi 2 \mathrm{P}$ two degrees of liberty were $0.964,0.985$ and 0.9803 for CAPN1-316, CAPN1-530 and CAST, respectively. According to the percentage of genotyping markers on capn 1 and CAST genes it concludes that the analyzed population has low levels of markers for softness or tenderness of the meat, probably by cross undirected carried out regularly, thus it aims to improve the quality of meat based on genetic programs improved using genetically superior animals resulting from this study.

Keywords: cattle dual purpose; meat tenderness; calpain; calpastatin. 


\section{Introducción}

El diagnóstico molecular se utiliza para apoyar los estudios genéticos asociados a enfermedades, tanto en humanos como en animales. Pero además, es también un conjunto de herramientas usadas para el análisis de marcadores moleculares en el genoma y proteoma de los individuos (Doelle et al., 2009), para detectar el potencial innato de los organismos como recursos locales para complementar las demandas alimentarias de las poblaciones humanas. En los países en desarrollo, esta tecnología ayuda a utilizar los recursos genéticos disponibles, a seleccionar aquellos portadores de genes asociados a características de importancia económica y a utilizarlos en cruzamientos dirigidos para generar una nueva estirpe que mejore la calidad de sus productos (FAO, 2010).

La tendencia actual del consumidor es demandar productos de buena calidad, por lo que, para mantener su competitividad, lleva a los productores de bovinos en el trópico a implementar estrategias de selección de progenitores que puedan transmitir estas características. Esta necesidad puede satisfacerse a través de la selección de características de importancia económica con determinaciones in vivo. Un ejemplo de lo anterior es el caso de los genes asociados a la calidad de la carne y la utilización molecular para su detección que conlleven a mejorar la ganadería de la región mediante cruzas dirigidas de los individuos genéticamente superiores. La falta de terneza en la carne es una causa de insatisfacción en el consumidor, y constituye uno de los criterios de selección más importantes para el consumidor a la hora de comprar carne (Brooks et al., 2000). Por lo que es una característica importante a mejorar en el ganado Cebú (Bos indicus) y sus cruzas.

Las calpaínas (CAPN) son proteasas que hidrolizan enlaces contiguos a la cisteína y que están implicadas en el proceso de maduración y enternecimiento de la carne (Goll et al., 2003). En el músculo esquelético existen dos isoformas principales: $\mu$-CAPN y m-CAPN. La calpastatina (CAST) es un inhibidor competitivo de dos proteasas dependientes de $\mathrm{Ca}^{2+}$, la $\mu$ CAPN y la m-CAPN (Eimori et al., 1988). El sistema CAPN/CAST está involucrado en el desarrollo del músculo (miogénesis), la comunicación entre células, la diferenciación celular y la degradación de proteínas musculares (Van den Maagdenberg et al., 2009) y de proteínas de la membrana celular durante la fusión de los mioblastos, al formarse el miotubo, y de células satélites (Moyen et al., 2004, Barnoy et al., 2005). Los estudios de estos procesos indican que 
pueden delinear el índice de producción y terneza de la carne en el animal adulto (Franco y Huttenlocher, 2005; Casas et al., 2006).

En el ganado de las especies Bos taurus y Bos indicus se han identificado varios polimorfismos de nucleótidos simples (SNP) en el gen $\mu$-CAPN, que se asociaron con la terneza o suavidad de la carne (Page et al., 2004; White et al., 2005; Casas et al., 2006). Por lo tanto, $\mu-$ CAPN es una proteasa con importancia económica por su efecto positivo sobre el grado de terneza de la carne (Geesink y Koohmaraie, 2000; Riley et al., 2003; Rickner et al., 2006).

White et al. (2005) evaluaron el efecto de cinco marcadores distribuidos a lo largo de todo el gen de $\mu$-CAPN y encontraron que dos SNP pueden ser utilizados para detectar una predisposición a variaciones en terneza en todas las especies de bovinos, incluyendo, bisontes (Bison bison y Bison bonasus) y yaks (Bos grunniens). Estos SNP fueron clasificados como CAPN1-316 y CAPN1-4751. Soria et al. (2010) reportaron otro SNP más asociado al mismo fenotipo, el CAPN1-530.

El polimorfismo CAPN1-316 se encuentra en el exón 9 que codifica a la región regulatoria de la proteína $\mu$-CAPN. Este SNP es una sustitución de citosina por guanina (alelos $\mathrm{C} \leftrightarrow \mathrm{G})$ que tiene como consecuencia el cambio del aminoácido 316 de alanína por glicina (Ala $\leftrightarrow$ Gly) (White et al., 2005). El polimorfismo CAPN1-530 provoca que se sustituya una adenina por una guanina (alelos $\mathrm{A} \leftrightarrow \mathrm{G}$ ) y que cambie el aminoácido 530 de isoleucina a valina (Soria et al., 2010). El alelo C, tanto en CAPN1-316 (Page et al., 2002 y 2004;) como en CAPN14751, se ha asociado con valores favorables de terneza de la carne, sin embargo, se ha encontrado en baja proporción en las poblaciones estudiadas (Page et al., 2002 y 2004; White et al., 2005; Casas et al., 2006).

En CAST se identificó un SNP de sustitución de citosina por timina (alelos $\mathrm{C} \leftrightarrow \mathrm{T}$ ) que está asociado a la terneza de la carne (Casas et al., 2006). Los animales homocigotos para timina (TT) producen carne con menores valores de fuerza de corte que aquellos homocigotos para citosina (CC) (Barendse, 2002). Schenkel et al. (2006) describieron la presencia de otro SNP en CAST que predispone a variaciones en la terneza similares a las descritas por Barendse (2002) y Casas et al. (2006). A diferencia de los estudios anteriores, el SNP de CAST donde se sustituye citosina por timina (alelos $\mathrm{C} \leftrightarrow \mathrm{T}$ ) también se consta de una sustitución de citosina por guanina (alelos $\mathrm{C} \leftrightarrow \mathrm{G}$ ). En este último SNP existe una menor fuerza de corte para el músculo Longissimus dorsi 7 d postmorten en los animales homocigotos para el alelo C (Bosques, 2007). 
Debido la importancia del sistema proteolítico CAST/CAPN1 y su relación con la terneza de la carne, el objetivo de este trabajo fue determinar la frecuencia de alelos asociados a la calidad de la carne en el ganado bovino de doble propósito de la región del Papaloapan.

\section{Materiales y Métodos}

\section{Animales}

Se utilizaron 331 bovinos de las cruzas Cebú, Criollo y razas europeas (Holstein, Pardo Suizo Americano, Pardo Suizo Europeo, Charolais, Beef Master y Angus) en la región del Papaloapan del estado de Oaxaca $\left(18^{\circ} 05^{\prime} \mathrm{N} 99^{\circ} 08^{\prime} \mathrm{W}, 20 \mathrm{~m}\right.$ altitud) que se usan para producir carne y leche, además de combinar el ordeño con el amamantamiento de los becerros hasta el destete. Los hatos se mantienen con pocos insumos y escaso uso de tecnología.

\section{Muestras de sangre}

Las muestras sanguíneas se colectaron de la vena caudal (5-10 $\mu \mathrm{L}$ /animal) y se depositaron en tubos (Vaccutainer) con EDTA como anticoagulante. Estas muestras se transportaron al Laboratorio de Biología Molecular de la Universidad del Papaloapan, Campus Tuxtepec, donde se almacenaron a $-20^{\circ} \mathrm{C}$ hasta su procesamiento.

\section{Extracción del ADN y amplificación de los productos de PCR}

El ADN se extrajo mediante el Kit de Extracción de ADN Sanguíneo GF-1 (Vivantis), usando la metodología indicada por el proveedor. Los marcadores moleculares CAPN1-316, CAPN1-530 y CAST se amplificaron mediante la Reacción en Cadena de la Polimerasa (PCR) con la polimerasa Dream Taq (Fermentas) a partir de $1 \mu \mathrm{L}$ de ADN genómico y 10 pM de los oligonucleótidos adecuados (Tabla 1) en un volumen final de reacción de $25 \mu \mathrm{L}$. La PCR se realizó en un termociclador (Maxygene, Axygen Scientific) con las siguientes condiciones de reacción: 1 ciclo $95{ }^{\circ} \mathrm{C} 3 \mathrm{~min} ; 35$ ciclos de $95{ }^{\circ} \mathrm{C}$ por $30 \mathrm{~s}$, la temperatura de alineamiento adecuada (Tabla 1) por $30 \mathrm{~s} \mathrm{y} 72{ }^{\circ} \mathrm{C}$ durante $30 \mathrm{~s}$; finalmente, 1 ciclo de $72{ }^{\circ} \mathrm{C}$ por $10 \mathrm{~min}$. Los productos de PCR $(10 \mu \mathrm{L})$ se sometieron a un análisis de electroforesis en gel de agarosa (2 $\%$ ) en amortiguador TAE $1 \mathrm{X}$ a $80 \mathrm{~V}$ por $40 \mathrm{~min}$. Las amplificaciones se observaron en un sistema de fotodocumentación (Ingenius, Syngenne). 
Tabla 1. Oligonucleótidos utilizados.

\begin{tabular}{lll}
\hline $\begin{array}{l}\text { Marcador } \\
\text { molecular }\end{array}$ & Secuencia & Ta $\left({ }^{\mathbf{0}} \mathbf{C}\right)$ \\
\hline CAPN1-316 & Directo: GAGCTGGCCCTCATAAGATAA & 58 \\
& Reverso: CCCATCCTCCATCTTGACC & \\
CAPN1-530 & Directo: GTGATTGTGCTGCGTTTCT & 62 \\
& Reverso: & \\
& AGAGACGGTGACAGCACCCTG & \\
DAST & Directo: & 58 \\
& AAGTAAAGCCAAAGGAACACACA & \\
& Reverso: AGGCTTTTTGGCTGAAAACA & \\
\hline
\end{tabular}

Ta: Temperatura de alineamiento.

\section{Genotipificación}

Se realizaron pruebas de RFLP para detectar a los marcadores (Tabla 2). CAPN1-316 se detectó con la enzima de restricción Btg I (CATALOGO, Fermentas), para el diagnóstico de los alelos C y G; CAPN1-530 con la enzima de restricción Ava II (CATALGO, Fermentas), para el diagnóstico de los alelos A y G; y CAST con la enzima de restricción Rsa I (Fermentas), para el diagnóstico de los alelos $\mathrm{C}$ y G. La digestión del producto amplificado con las enzimas de restricción se realizó con $15 \mu \mathrm{L}$ del volumen de la reacción de PCR, $2.5 \mu \mathrm{L}$ de 10X Buffer, 6.5 $\mu \mathrm{L}$ de agua estéril y $10 \mathrm{U}$ de la enzima correspondiente. La mezcla se incubó por 12 horas a 37 ${ }^{\circ} \mathrm{C}$ en un horno de convección. Los fragmentos digeridos se separaron por electroforesis en gel de agarosa (3\%) en buffer TAE 1X como se describió anteriormente. La lectura de alelos se realizó calculando el tamaño de los fragmentos con a un marcador de 50 pb (Fermentas). 
Tabla 2. Ubicación genómica y patrón de restricción esperado de los marcadores moleculares CAPN1-316, CAPN1-530 y CAST.

\begin{tabular}{|c|c|c|c|}
\hline \multirow{2}{*}{ Característica } & \multicolumn{3}{|c|}{ Marcador molecular } \\
\hline & CAPN1-316 & CAPN1-530 & CAST \\
\hline Secuencia en GenBank & AF252504 & AF248054 & AY008267.1 \\
\hline \multirow[t]{2}{*}{ Localización genómica } & Cromosoma & Cromosoma & Cromosoma \\
\hline & Exón 9 & Exón 14 & Exón 5 \\
\hline Producto de PCR (pb) & 218 & 460 & 434 \\
\hline Enzima de restricción & Btg I & Ava II & Rsa I \\
\hline \multirow[t]{2}{*}{ Posiciones de corte } & Alelo G: no corta & Alelo A: 44, 226 & Alelo C: no corta \\
\hline & Alelo C: 169 & Alelo G: 44, 166 & Alelo G: 255 \\
\hline \multirow{4}{*}{$\begin{array}{l}\text { Patrón de restricción esperado de } \\
\text { acuerdo al alelo }(\mathrm{pb})\end{array}$} & GG: 218 & AA: $44,182,234$ & CC: 434 \\
\hline & CC: 169,49 & GG: 44, 122, 294 & GG: 168,255 \\
\hline & GC: $218,169,49$ & AG: $44,122,182$, & CG: $434, \quad 255$, \\
\hline & & 294 & 168 \\
\hline
\end{tabular}

\section{Análisis estadístico}

Para el análisis estadístico de la variabilidad genética de CAPN1 y CAST se calcularon las frecuencias alélicas y genotípicas, y el equilibrio Hardy Weimberg con la prueba de $\chi^{2}$ (Ji

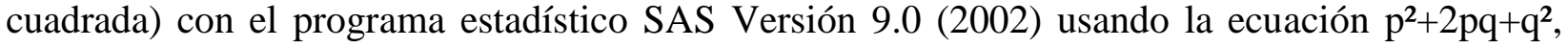
donde:

$\mathrm{p}^{2}=$ alelos en individuos homocigotos con un polimorfismo $n$

$2 \mathrm{pq}=$ frecuencia predicha para heterocigotos

$\mathrm{q}^{2}=$ alelos en individuos homocigotos con un polimorfismo $m$

\section{Resultados}

De los 331 animales a los que se les extrajo ADN genómico, únicamente en 272 se logró amplificar productos de PCR para al menos uno de los 3 marcadores. La falta de amplificación de varias muestras en cada uno de los marcadores, puede ser un reflejo de que el sistema de genotipificación empleado puede no funcionar con el tipo de cruzas que se encuentra en la Cuenca, por lo que es necesario profundizar posteriormente en esta investigación, tal y como lo 
menciona Casas et al. (2005), en donde observaron que en tres hatos de bovinos, este sistema sólo fue exitoso para dos, siendo el restante diferente por poseer mayor "carga" genética de Bos indicus. Mientras que para los individuos que sí se pudo amplificar con el sistema de genotipificacion usado, se realizó RFLP y cuantificación de discriminación alélica (Casas et al 2006).

\section{Polimorfismo CAPN1-316}

El producto de PCR del marcador CAPN1-316 se amplificó a partir de ADN genómico y se observó el producto esperado de 218 pb. Después de la digestión con la enzima BtgI se observaron los patrones de restricción esperados de 169 y 49 pb para el genotipo CC, un fragmento de 218 pb para los individuos GG y 218, 169 y 49 pb en los individuos GC.

Para CAPN1-316 se observó que el genotipo de mayor frecuencia fue GG (86 \%) seguido por CG $(11 \%)$, y CC el menos común (3\%). El alelo C constituyó un $14 \%$ del total, mientras que el $\mathrm{G}$ un $86 \%$. El alelo $\mathrm{C}$, ha sido asociado con valores favorables de terneza de la carne, sin embargo, se ha encontrado una baja proporción de este en las poblaciones de ganado para carne estudiadas (Page et al., 2002 y 2004; White et al., 2005; Casas et al., 2006). La heterocigosidad media fue de 0.114 (Tabla 3).

Tabla 3. Frecuencias genotípicas y alélicas del marcador molecular CAPN1-316, observadas y esperadas en bovinos de doble propósito.

\begin{tabular}{|l|l|l|l|l|l|}
\hline \multicolumn{2}{|l|}{ Observado } & \multicolumn{2}{l|}{ Esperado $\left(\mathbf{p}^{2}+\mathbf{2 p q}+\mathbf{q}^{2}\right)$} \\
\hline Genotipo & $\begin{array}{l}\text { Número de } \\
\text { individuos }\end{array}$ & $\begin{array}{l}\text { Frecuencia } \\
\text { genotípica }\end{array}$ & $\begin{array}{l}\text { Frecuencia } \\
\text { genotípica }\end{array}$ & Alelos & $\begin{array}{l}\text { Frecuencia } \\
\text { alélica }\end{array}$ \\
\hline CC & 3 & 0.03 & 0.01 & G & 0.86 \\
\hline GG & 90 & 0.86 & 0.84 & C & 0.14 \\
\hline CG & 12 & 0.11 & 0.16 & & \\
\hline Heterocigosidad $\mathbf{0 . 1 1 4}$ & & & & \\
\hline
\end{tabular}

\section{Polimorfismo CAPN1-530}

El producto de PCR de CAPN1-530 fue de 460 pb, cuando se digirió con la enzima AvaII, se observaron los patrones de restricción 44, 182 y 234 pb para el genotipo AA; 44, 122 y 294 pb 
para individuos GG y 44,122, 182, y 294 pb para GA (Figura 2). En el análisis de CAPN1-530 se observó una frecuencia genotípica en la población global de 2, 85 y $13 \%$, para AA, GG y AG respectivamente (Tabla 4). El alelo A es el favorable para la suavidad de la carne, sin embargo solo represento el $15 \%$ del total, mientras que el $\mathrm{G}$ formó $85 \%$, con una heterocigosidad de 0.156 .

Tabla 4. Frecuencias genotípicas y alélicas de CAPN1-530 observadas y esperadas en bovinos de doble propósito.

\begin{tabular}{|l|l|l|l|l|l|}
\hline \multicolumn{2}{|l|}{ Observado } & \multicolumn{2}{l|}{ Esperado $\left(\mathbf{p}^{2}+\mathbf{2 p q}+\mathbf{q}^{2}\right)$} \\
\hline Genotipo & $\begin{array}{l}\text { Número de } \\
\text { individuos }\end{array}$ & $\begin{array}{l}\text { Frecuencias } \\
\text { Genotípicas }\end{array}$ & $\begin{array}{l}\text { Frecuencias } \\
\text { Genotípicas }\end{array}$ & Alelos & $\begin{array}{l}\text { Frecuencias } \\
\text { Alélicas }\end{array}$ \\
\hline AA & 1 & 0.02 & 0.01 & A & 0.15 \\
GG & 40 & 0.85 & 0.84 & G & 0.85 \\
AG & 6 & 0.13 & 0.15 & & \\
\hline \multicolumn{4}{|l|}{ Heterocigosidad } & $\mathbf{0 . 1 5 6}$ & \\
\hline
\end{tabular}

\section{Polimorfismos de CAST}

El producto de PCR del marcador CAST fue de 434 pb. El producto se digirió con la enzima RsaI y se observaron los patrones de restricción 168 y 255 pb en los individuos GG, sin corte en los individuos que presentan el genotipo CC, y 434, 255 y 168 pb en los individuos GC. En CAST se observó una frecuencia genotípica global para CC de $32 \%$, CG de $40 \%$ y GG 28 \% (n=120; Tabla 5). La figura 10 muestra la frecuencia de los alelos $\mathrm{C}$ con el $32 \%$ y el alelo $\mathrm{G}$ el $68 \%$. Donde el alelo favorable es el C, mostrando un porcentaje aceptable dentro de la población. Asimismo, se observa la heterocigosidad media es de 0.499 de los mismos. 
Tabla 5. Frecuencias genotípicas y alélicas de CAST observadas y esperadas en bovinos de doble propósito.

\begin{tabular}{|l|l|l|l|l|l|}
\hline \multicolumn{2}{|l|}{ Observado } & \multicolumn{2}{l|}{ Esperado $\left(\mathbf{p}^{2}\right.$ 2pq q } \\
\hline Genotipo & $\begin{array}{l}\text { Número de } \\
\text { individuos }\end{array}$ & $\begin{array}{l}\text { Frecuencias } \\
\text { Genotípicas }\end{array}$ & $\begin{array}{l}\text { Frecuencias } \\
\text { Genotípicas }\end{array}$ & Alelos & $\begin{array}{l}\text { Frecuencias } \\
\text { Alélicas }\end{array}$ \\
\hline CC & 38 & 0.32 & 0.27 & G & 0.68 \\
GG & 34 & 0.28 & 0.23 & C & 0.32 \\
CG & 48 & 0.4 & 0.5 & & \\
\hline \multicolumn{7}{|l|}{ Heterocigosidad } & $\mathbf{0 . 4}$ & \\
\hline
\end{tabular}

\section{Discusión}

La ganadería del trópico mexicano enfrenta diversos retos, tal como las condiciones climáticas adversas, forrajes de baja calidad, escaso uso de registros productivos y reproductivos, y poco uso de tecnologías de selección genética avanzada (Cortés-López et al., 2012), que complementen el uso de herramientas tradicionales de mejoramiento genético animal. Cuando una empresa ganadera tienen los objetivos de producción claros, los métodos de análisis molecular genéticos pueden auxiliar a enfocarse hacia lo que se quiere y trabajar en el mejoramiento de dichas caracerísticas, ya sea leche o carne (Van Eenennaam et al., 2014). Por ejemplo, en el caso de que el objetivo sea la producción de carne, se deberán de enfocar las estrategias de mejoramiento genético con base en la introducción de ganado europeo que proporcione esta característica, a la que se le dará seguimiento molecular, y así lograr a largo plazo el mejoramiento genético de los animales.

En este trabajo se utilizaron 331 bovinos de las cruzas Cebú, Criollo y razas europeas en la región del Papaloapan del estado de Oaxaca que se usan para producir carne y leche. Los hatos se mantienen con pocos insumos y escaso uso de tecnología. La información genómica y el uso de algunas de sus herramientas como la PCR y las enzimas de restricción para la genotipificación, se pueden usar en complemento con la información genealógica e información fenotípica de los animales, para el diseño de estrategias de selección genética y mejorar así las características de importancia económica como son, la calidad de la leche y el grado de terneza o 
suavidad de la carne para de esta manera ofertar al consumidor un producto de mejor calidad o de aquellas características que requieran el sacrificio del animal o de expresión en un solo sexo. La identificación de características genéticas deseables puede brindar una forma rápida de diagnóstico molecular sobre los QTLs relevantes en la industria pecuaria y así, diseñar estrategias de mejoramiento genético dirigido que acompañen a los métodos tradicionales de selección y cruzamiento.

Diversos estudios han demostrado que variaciones genotípicas de $\mu$-CAPN, m-CAPN y su inhibidor CAST están relacionadas con características de importancia económica como la terneza de la carne (Barendse et al., 2002; Morris et al., 2006) y se han propuestos como marcadores moleculares (Casas et al., 2006). Por ello, en el presente trabajo se determinaron las frecuencias genotípicas y alélicas de los polimorfismos CAPN1-316, CAPN1-530 y CAST en ganado de doble propósito en la región cuenca del Papaloapan en el estado de Oaxaca.

\section{Frecuencia de CAPN-316}

El sistema CAPN1/CAST también está ligado con el desarrollo celular en etapas pre- y posnatales de manera positiva (Casas et al., 2006; Chung et al., 2007; Van den Maagdenberg et al., 2009). El polimorfismo CAPN1-316 está relacionado con variaciones en las características de ganancia en peso vivo y peso a la matanza en animales de las razas Angus y Brangus criados bajo pastoreo y sacrificados a un espesor de grasa dorsal promedio de $6.0 \mathrm{~mm}$ (Miquel et al., 2009).

En los estudios realizados por Page et al. (2002 y 2004), White et al. (2005) y Casas et al. (2005 y 2006) se identificaron polimorfismos que afectan la morfología de la proteína de CAPN1 de tal modo que su acción podría variar a medida que cambia la base nitrogenada. Page et al. (2004) describieron dos polimorfismos responsables de variación del gen de $\mu$-calpaina. Ambos polimorfismos han sido intensamente estudiados en varias poblaciones y subespecies bovinas (White et al., 2005). El resultado de estas investigaciones demuestra que en todos los genotipos de CAPN1-316 se relacionan con variaciones en terneza de la carne de todas las subespecies bovinas, mientras que CAPN1-530 ejerce un efecto similar solamente en el ganado Bos taurus.

Los resultados de esta investigación muestran que para el marcador CAPN1-316 abunda principalmente el genotipo GG con un $86 \%$, mientras que el genotipo CC es del $3 \%$ y el heterocigoto CG es del $11 \%$, estos resultados son similares a lo encontrado por Leal-Gutiérrez et al. (2015) en cruzas de Bos taurus y Bos indicus, donde reportan genotipos GG, CC y CG y, con

No 19, Vol. 9 (2), 2017. ISSN 2007 - 0705, pp.: 211 - 228 
$91 \%, 0 \%, 9 \%$, respectivamente. Mientras que la frecuencia alélica de $\mathrm{G}$ fue mayor que el alelo C con 86 y $14 \%$, respectivamente. Estas frecuencias son similares a lo reportado previamente (Page et al., 2004; White et al., 2005; Casas et al., 2006; Corva et al., 2007; Chung et al., 2007, Van den Magdenberg et al., 2009). Debido a que el genotipo CC está asociado directamente a la suavidad de la carne (Barendse, 2002; Casas et al., 2006), se concluye que la mayoría de los animales usados en esta investigación tienen bajos índices de suavidad en su carne. Esto muestra que no ha habido un programa dirigido hacia razas especializadas en la producción de carne, sino más bien a razas lecheras; sin embargo, es necesario establecer un programa de mejora genética basado en la identificación de animales genéticamente superiores, en producción de carne, para posteriormente incorporarlos en programas de mejoramiento genético en los hatos ganaderos de la región y mejorar la suavidad de la carne.

La metodología usada en este trabajo permitió identificar a 10 individuos deseables (CC) en un hato de 331 cabezas, esta información puede ser usada en conjunto con caracteres como velocidad del crecimiento, rendimiento de la canal, conformación muscular para implementar un programa controlado de cruzas que también puede ir acompañado de mediciones de fuerza de corte para el músculo de cada genotipo y un análisis comparativo transgeneracional.

\section{Frecuencia de CAPN-530}

Para el marcador CAPN1-530, el alelo G fue más común con una frecuencia alélica del $85 \%$, mientras que el alelo A solo presentó una frecuencia alélica del $15 \%$. En cuanto a las frecuencias genotípicas para AA, GG y AG, presentaron 2, 85, y $13 \%$, respectivamente. Leal-Gutiérrez et al. (2015), reportaron 0.40 y $60 \%$ respectivamente, diferencias significativas con respecto a este estudio, debido posiblemente a la gran variación entre razas existentes en nuestra población y a que en cada región existe una mezcla distinta de animales, aunado al nulo registro de parámetros tanto productivos como reproductivos, para tener una visión clara de lo que ocurre en las explotaciones ganaderas, lo que indica la necesidad de llevar a cabo estos análisis en cada unidad productiva.

Aunado a lo anterior, los objetivos de producción no están bien definidos por los productores de ganado bovino, lo que puede limitar la validación de un mismo marcador y su asociación a un fenotipo determinado, dados los efectos ambientales y genéticos diferenciales. Por otro lado, Casas et al. (2006), Van Eenennaam et al. (2007), Allais et al. (2011) y Li et al. 
(2010) encontraron que en el marcador CAPN1-530, los individuos con genotipo AA presentaban carne más blanda respecto a animales AG y GG. Nuevamente, esto indica que los métodos tradicionales de crianza de ganado no favorecen de forma eficiente el aumento de las poblaciones con esta característica.

Page et al. (2004), reportaron que el marcador CAPN1-316 tiene más capacidad de predicción en la población de sementales americanos, pero el marcador CAPN1-530 puede ser más común en otras poblaciones de animales. En este estudio, se encontró que el marcador más abundante fue el CAPN1-316, mismo que es reportado por Page et al. (2004) como un marcador de mejor predicción para animales como mayor terneza. En este estudio no predomino el alelo C, lo que tendría como consecuencia que los índices de terneza no sean los mejores que se pueden obtener. Por otro lado, CAPN1-530 se ha reportado como el marcador más abundante en Bos taurus (Page et al., 2004), y en esta investigación, fue el que menos se encontró, esto indica que en las poblaciones de ganado bovino de doble propósito de la región del Papaloapan predomina el ganado Bos indicus, y posiblemente no se presente un buen índice de terneza, de acuerdo con los resultados obtenidos.

\section{Frecuencias de CAST}

Para CAST se encontró una frecuencia alélica en $\mathrm{C}$ del $32 \%$ y el alelo $\mathrm{G}$ del $68 \%$, a pesar de que el alelo $\mathrm{C}$ presenta una menor frecuencia, es el asociado con una menor fuerza de corte, lo que significa que presenta una mayor suavidad de la carne. Motter et al. (2009) reportaron valores de corte en CAST de $6.73 \pm 0.15,6.99 \pm 0.13$ y $7.04 \pm 0.22 \mathrm{~kg}$ para los genotipos CC, CG y GG, respectivamente, y la diferencia entre CC y CG $(-0.26 \mathrm{~kg})$ presentó un valor cercano a la significancia estadística $(\mathrm{p}=0,06)$.

Por otro lado, Leal y Jiménez (2015) establecieron diferencias in-silico en la estabilidad molecular del ARNm del alelo G del marcador CAST, cuando se comparó con el del alelo A del marcador CPN1-530. Teóricamente, las diferencias en estabilidad podrían suministrar al ARNm del alelo G una mayor estabilidad, permitiendo una mayor expresión de la proteína CAST y por lo tanto una mayor inhibición de la proteína CAPN1, lo que originaría carnes más duras (Casas et al., 2006; Van Eenennaam et al., 2007; Li et al., 2010; Allais et al., 2011). 
Para el alelo C, tanto del marcador CAPN1-316 y CAST, así como para el alelo A del CAPN1-530, es importante realizar cruzas dirigidas para mejorar y perpetuar la estirpe con la finalidad de aumentar la calidad de la terneza en carne.

A partir de estos datos se sugiere hacer pruebas correspondientes de correlación de genotipo e indicadores fenotípicos, con la finalidad de seleccionar a aquellos individuos con características deseables para la suavidad de la carne.

\section{Conclusiones}

Se determinaron las variantes genéticas de los genes CAPN1 y CAST en 331 animales de doble propósito, donde el genotipo $\mathrm{CC}$ de $C A P N 1-316$ y $C A S T$, que ha sido asociado a mayor terneza de la carne, ha sido el de menor frecuencia. Mientras que para CAPN1-530, predominó el homocigoto GG, sin embargo, el genotipo AA presenta carne más blanda con respecto a los demás genotipos. La alta frecuencia de alelos G, en los tres marcadores, en contraste con la casi nula frecuencia de homocigotos $\mathrm{C}$, A y $\mathrm{C}$, en los marcadores CAPN1-316, CAPN1-530 y CAST, respectivamente, se debe al tipo de ganado de la región, cruzas entre razas lecheras y cebuinas, se esperaban bajos resultados para los alelos favorables y a la poca orientación hacia un objetivo de producción. De acuerdo con el porcentaje de genotipos resultantes de los genes CAPN1 y CAST, la población analizada tiene una alta probabilidad de expresar bajos índices de suavidad o terneza de la carne. De tal forma, se concluye que si los productores desean aumentar la calidad de la carne en sus hatos, es necesario introducir estos polimorfismos mediante un programa de selección genética asistida con las herramientas moleculares aquí empleadas. Con la aplicación de estos avances, se beneficiaría a los productores de ganado y por lo tanto, el estatus de la industria del ganado para carne en el trópico mexicano.

\section{Referencias}

Allais, S., L. Journaux, H. Levéziel, N. Payet-Duprat, J.F. Hocquette, J. Lepetit, S. Rousset, C. Denoyelle, C. Bernard-Capel, and G. Renand. (2011). Effects of polymorphisms in the calpastatin and $\mu$-calpain genes on meat tenderness in 3 French beef breeds. J. Anim. Sci. 89:1-11.

Barendse, W.J. (2002). DNA markers for meat tenderness. International patent application PCT/AU02/00122. International patent publication WO 02/064820 A1.

No 19, Vol. 9 (2), 2017. ISSN 2007 - 0705, pp.: 211 - 228 
Barnoy, S., M. Maki, and N.S. Kosower. (2005). Overexpression of calpastatin inhibits L8 myoblast fusion. Biochem. Biophys. Res. 332(3): 697-701.

Bosques, M.J.H. (2007). Segregación de polimorfismos identificados en los genes de $\mu$-calpaina y calpastatina y su relación con el crecimiento corporal y características de la canal de bovinos para carne en Puerto Rico. Tesis de Maestría, Universidad de Puerto Rico. Puerto Rico.

Brooks, J.C., J.B. Belew, D.B. Griffin, B.L. Gwartney, D.S. Hale, W.R. Henning, D.D. Johnson, J.B. Morgan, F.C. Parrish Jr, J.O. Reagan, and J.W. Savell. (2000). National Beef Tenderness Survey-1998. J Anim Sci. 78(7):1852-1860.

Casas, E., S.N. White, D.G. Riley, T.P.L. Smith, R.A. Brennemant, T.A. Olson, D.D. Johnson, S.W. Coleman, G.L. Bennett and C.C. Chase Jr. (2005). Assessment of single nucleotide polymorphisms in genes residing on chromosomes 14 and 29 for association with carcass composition traits in Bos indicus cattle. Journal of Animal Science, 83(1): 13-19.

Casas, E., S.N. White, T.L. Wheeler, S.D. Shackelford, M. Koohmaraie, D.G. Riley, C.C. Chase Jr., D.D. Johnson and T.P.L. Smith. (2006). Effects of calpastatin and $\mu$-calpain markers in beef cattle on tenderness traits. J. Anim. Sci. 84(3):520-525.

Chung H.Y., M.E. Davis, and H.C. Hines. (1999). A DNA polymorphism of the bovine calpastatin gene detected by SSCP analysis. Anim. Genet. 30(1): 80-81.

Cortés-López, N.G., S. del Moral, J.A. Rueda, C. Luna-Palomera, C.A. Meza-Herrera and J. Abad-Zavaleta. (2012). Allelic and genotypic frequency of kappa casein gene in double purpose cattle. Tropical and Subtropical Agroecosystems, 15: 47-55.

Corva, P.M., L. Soria, A. Schor, E.Villarreal, M.P. Cenci, M. Motter, C. Mezzadra, L. Melucci, C. Miquel, E. Pavònn, G. Depetris, F. Santini and J.C. Naìn. (2007). Association of CAPN1 and CAST gene polymorphisms with meat tenderness in Bos taurus beef cattle from Argentina. Genetics and Molecular Biology, 30(4): 1064-1069.

Doelle, W., S. Rokem, M. Berovic. (2009). In: Methods in biotechnology, Vol. 2, Ed. Horst.

Eimori, Y., H. Kawasaki, S. Imajoh, Y. Minami and K. Suzuki. (1988). All four repeating domains of the endogenous inhibitor for calcium-dependent protease independently retain inhibitory activity. Expression of the cDNA fragments in Escherichia coli. J Biol Chem. 263:2364-2370. 
FAO. (2010). La situación de los recursos zoogenéticos mundiales para la alimentación y la agricultura.

Geesink G.H. and M. Koohmaraie. (2000). Ionic strength-induced inactivation of $\mu$-calpain in postmortem muscle. J. Anim. Sci. 78: 2336-2343.

Goll D.E., V.F. Thompson H. Li, W. Wei, y J. Cong. (2003). The calpain system. Physiol. Rev. 83(3): 731-801.

Leal-Gutiérrez, J.D., L.M. Jiménez-Robayo, M. Ariza, C. Manrique, J. López, C. Martínez, Y. Pinilla, S. Castro, N. García, C. Bedoya, A. Jiménez. (2015). Polimorfismos de los genes CAPN1, CAST, DES, PRKAG3 y RYR1 asociados a la capacidad de retención de agua en crudo y cocinado en carne de bovino en cruces Bos indicus y Bos taurus en Colombia. Arch. Zootec. 64 (245): 29-35.

Li, J., L.P. Zhang, Q.F. Gan, J.Y. Li, H.J. Gao, Z.R. Yuan, X. Gao, J.B. Chen, and S.Z. Xu,. (2010). Association of CAST gene polymorphisms with carcass and meat quality traits in Chinese commercial cattle herds. Asian-Australasian Journal of Animal Sciences, 23(11): $1405-1411$.

Miquel, M.C., E. Villarreal, C. Mezzadra, L. Melucci, L. Soria, P. Corva and A. Schor. (2009). The association of CAPN1316 marker genotypes with growth and meat quality traits of steers finished on pasture. Genetics and Molecular Biology, 32 (3), pp. 491-496.

Morris, C.A., N.G. Cullen, S.M. Hickey, P.M. Dobbie, B.A. Veenvliet, T.R. Manley, W.S. Pitchford, Z.A. Kruk, C.D.K. Bottema and T. Wilson. (2006). Genotypic effects of calpain 1 and calpastatin on the tenderness of cooked M. longissimus dorsi steaks from Jersey x Limousin, Angus and Hereford-cross cattle. Animal Genetics, 37(4): 411-414.

Motter, M.M., P. Corva, M. Krause, M. Perez-Cenci and L. Soria. (2009). Role of calpastatin in the variability of beef tenderness. Journal of Basic and Applied Genetics, 20(1): 15-24.

Moyen C., S. Goudenege, S. Poussard, A.H. Sassi, J.J. Brustis, and P. Cottin. 2004. Involvement of micro-calpain (CAPN 1) in muscle cell differentiation. Int. J. Biochem. \& Cell Biol. 36(4): 728-743.

Page, B.T., E. Casas, M.P. Heaton, N.G. Cullen, D.L. Hyndman, C.A. Morris, A.M. Crawford, T.L. Wheeler, M. Koohmaraie, J.W. Keele and T.P. Smith. (2002). Evaluation of singlenucleotide polymorphisms in CAPN1 for association with meat tenderness in cattle. J. Anim. Sci. 80(12):3077-3085. 
Page, B.T., E. Casas, R.L. Quaas, R.M. Thallman, T.L. Wheeler, S.D. Shackelford, M. Koohmaraie, S.N. White, G.L. Bennett, J.W. Keele, M.E. Dikeman and T.P. Smith. (2004). Association of markers in the bovine CAPN1 gene with meat tenderness in large crossbred populations that sample influential industry sires. J. Anim. Sci. 82(12): 34743481 .

Ricnker, C.B., N.A. Pyatt, L.L. Berger, and D.B. Faulkner. (2006). Relationship among GeneSTAR marbling marker, intramuscular fat deposition, and expected progeny difference in early weaned Simmental steers J. Anim. Sci. 84(3): 686-693.

Riley, D.G., C.C. Chase Jr., T.D. Pringle, R.L. West, D.D. Johnson, T.A. Olson, A.C. Hammond and S.W. Coleman. (2003). Effect of sire on $\mu$ and m-calpain activity and rate of tenderization as indicated by myofibril fragmentation indices of steaks from Brahman cattle. J. Anim. Sci. 81(10):2440-2447.

Santos J.F. and A. Huttenlocher. (2005). Regulating cell migration: calpains make the cut. J. Cell Sci. 118: 3829-3838.

SAS Institute. (2002). SAS User's Guide: Statistics. Version 9.0. Statistic Analysis System Institute. Cary, North Carolina, USA. 132p.

Schenkel F.S., S.P. Miller, Z. Jiang, I.B. Mandell, X. Ye, H. Li, and J. W. Wilton. (2006). Association of a single nucleotide polymorphism in the calpastatin gene with carcass and meat quality traits of beef cattle. J. Anim. Sci. 84(2): 291-299.

Soria L.A., P.M. Corva, M.J. Huguet, S. Miño and M.C. Miquel. (2010). Bovine $\mu$-calpain (capn1) gene polymorphisms in Brangus and Brahman bulls. Journal of Basic \& Applied Genetics, 21(1): 61-69

Van den Maagdenberg, K., E. Claeys, A. Stinckens, N. Buys and S. De Smet. (2007). Effect of age, muscle type, and insulin-like growth factor-II genotype on muscle proteolytic and lipolytic enzyme activities in boars1. Journal of Animal Science 85 (4): 952-960.

Van Eenennaam, A.L., J. Li, R.M. Thallman, R.L. Quaas, M.E. Dikeman, C.A. Gill, D.E. Franke and M.G. Thomas. (2007). Validation of commercial DNA tests for quantitative beef quality traits. Journal of Animal Science, 85(4): 891-900.

Van Eenennaam, A.L., Weigel, K.A., Young, A.E., Cleveland, M.A., Dekkers, J.C.M. (2014). Applied animal genomics: Results from the field. Ann. Rev. Anim. Biosci. 2(105-139). 
White, S.N., E. Casas, T.L. Wheeler, S.D. Shakelfold, M. Koohmaraie, D.G. Riley, C.C. Chase, Jr., D.D. Johnson, J.W. Keele and T.P.L. Smith. (2005). A new single nucleotide polymorphism in CAPN1 extends the current tenderness marker test to include cattle of Bos indicus, Bos taurus, and crossbred descent. J. Anim. Sci. 83(9): 2001-2008. 